\title{
Synthesis of Sorbitan Ester Stabilized Uniform Spherical Silver Nanoparticles
}

\author{
PRATEEK SHUKLA ${ }^{1 *}$, TANDRA NANDI ${ }^{2}$ and RAJINDER PAL SINGH ${ }^{3}$ \\ 'Department of Chemistry, Indian Institute of Technology, Kanpur- 208016, India. \\ 2Defence Materials and Stores Research and Development Establishment, \\ G. T. Road, Kanpur-208013, India. \\ ${ }^{3}$ Department of Oil and Paint Tech., H. B. Technological Institute, Kanpur- 208002, India. \\ ${ }^{*}$ Corresponding author E-mail: prateek.shukla8@gmail.com \\ http://dx.doi.org/10.13005/ojc/320614
}

(Received: July 04, 2016; Accepted: October 02, 2016)

\begin{abstract}
Controllable synthesis of uniform spherical silver nanoparticles was investigated by carrying out a chemical reduction reaction in a micro emulsion, formed under high shear mixing of aqueous silver nitrate solution and 2-ethyl hexanol as organic phase. Sodium borohydride was used as reducing agent and sorbitan monooleate (span-80) as stabilizer. Further, a study has been performed to observe the effect of silver nitrate concentration, reducing agent concentration, amount of surfactant and organic phase on the size and shape of silver nanoparticles. Transmission electron microscopy (TEM) and dynamic light scattering (DLS) techniques were used for elucidating the shape, size and size distribution of the synthesized silver nanoparticles. Crystal structure of silver nanoparticles thus prepared has been studied by X-ray diffraction analysis (XRD). Uniform silver nanoparticles of average diameter 15-20 nm with high purity and well crystalline nature were obtained under optimized set of conditions. The results of synthesis of these nanoparticles in present study were excellent and may also be used at larger scale.
\end{abstract}

Keywords: silver nanoparticles, DLS, TEM, micro emulsion, XRD, span-80.

\section{INTRODUCTION}

In recent years, a great interest in metal nanoparticles with controlled size and size distribution has been observed. Because of unusual properties of nanostructured silver particles, these nanoparticles have been the subject of numerous intensive researches. In comparison with larger particles, these exhibit new/ improved properties (e.g. higher surface to volume ratio, changes in total energy, changes in conductivity, different optical features) ${ }^{1}$. To prepare silver nanoparticles with specific size and size distribution has thus become an essential research area in recent years.Many methods have been exploited for the synthesis of silver nanoparticles in the past few years; where- 
in chemical reaction in micro emulsion is one of the most important methods for synthesis of monodisperse nanoparticles ${ }^{2}$.

Chemical reduction method can be considered suitable for the production of large quantities of nanoparticles in relatively shorter period of time ${ }^{3}$. A typical synthesis of silver nanoparticles by chemical reduction process consists of three main stages. The first one is the reduction of a silver salt by a reducing agent. $\mathrm{AgNO}_{3}$ is most frequently used as a source of silver ions and $\mathrm{NaBH}_{4}$ as a reducing agent. In the second stage, neutral silver atoms collide with each other, forming stable nuclei and a growth of the nanoparticle occurs until all metals ions are consumed. The final stage involves prevention of nanoparticles agglomeration by the addition of protecting agents. Stabilizers prevent the agglomeration via their interaction with small particles ${ }^{4,5}$. Moreover, by chemical reduction method, morphology (i.e. diameter, size distribution, shape) of nanostructured silver nanoparticles can be controlled by various parameters. The important key in this approach is to avoid the agglomeration of silver nanoparticles during the synthesis and preservation procedure. Usually special organic compounds such as surfactants are used for the purpose. Depending on the proportions of various components employed, a surfactant with specific hydrofilic-lypofilic balance (HLB) value is used. The microdroplets in the microemulsion can be in the form of oil-swollen micelles dispersed in an aqueous phase as o/w microemulsion or water-swollen micelles dispersed in oil phase as w/o microemulsion ${ }^{6,7,8,9}$.

Nanoemulsions are non-equilibrium systems and cannot be formed spontaneously. They can be produced using two different approaches: high energy and low energy methods. High energy methods use intense mechanical forces to break up macroscopic phases or droplets into smaller droplets and typically involve the use of mechanical devices known as homogenizers, which may use high-shear mixing, high-pressure homogenization or ultrasonification. In contrast low-energy methods rely on the spontaneous formation of emulsion under specific system compositions or environmental conditions as a result of changes in interfacial properties. Spontaneous emulsification is a less expensive and energy efficient alternate that takes advantage of the chemical energy stored in the system. High energy methods are effective in reducing droplet size and with the aid of proper stabilizer called surfactants. Surfactants can be employed effectively for the synthesis of various nanoparticles 10,11,12. Generally, sodium borohydride, hydrazine, ascorbic acid and cetyltrimethylammonium bromide, sodium dodecyl sulphate, poly (vinyl alcohol), poly (vinylpyrrolidone), are used as the reducing- and stabilizing-agents respectively to prepare silver nanoparticles with controlled size and shape ${ }^{13}$.

In the present paper we have described an efficient and cost effective method for production of large quantity of uniform silver nanoparticles using high energy microemulsion method. Silver nitrates as silver ion source, sodium borohydride as reducing agent, 2- ethyl hexanol as organic phase and span-80 as stabilizer have been used. Further, investigations have also been carried out to see the effect of silver salt concentration, strength of reducing agent, concentration of stabilizer and amount of organic phase on the size of silver nanoparticles along with their particle size distribution. Transmission electron microscopy (TEM) and dynamic light scattering (DLS) techniques were used for elucidating the shape, size and size distribution of the synthesized silver nanoparticles. Crystal structure of silver nanoparticles thus prepared has been studied by $\mathrm{X}$-ray diffraction analysis (XRD).

\section{MATERIALS AND METHOD}

A synthetic method developed for the purpose of this experiment enables the production of stable silver nanoparticles under optimized set of conditions. The silver nitrate $\left(\geq 99.0 \%, \mathrm{AgNO}_{3}\right)$ and sodium borohydride $\left(\geq 96.0 \%, \mathrm{NaBH}_{4}\right)$ were purchased from Sigma-Aldrich and Merck chemicals respectively. Span-80 (non-ionic, HLB= 4.3) and 2-ethyl hexanol were purchased from Fluka chemicals. All chemicals were used without further purification. Double distilled water was used for the preparation of all samples.

\section{Synthesis of Silver nanoparticles}

Synthesis of silver nanoparticles by chemical reduction method using silver nitrate as a source of silver ion and sodium borohydride as reducing agent have been used by many researchers. However 
fewer claimed their work suitable for bulk synthesis with controlled particle size distribution. A. Katiyaret al. have reported synthesis of Fe-Ni nanoparticles with controlled morphology for preparing magnetic nanofluid ${ }^{14}$. In the present study, span-80 stabilized spherical silver nanoparticles were synthesized via forming a microemulsion by high energy methods ${ }^{11}$. Here microemulsion is formed by mixing bulk amount $(250 \mathrm{ml})$ of aqueous silver nitrate solution (as water phase), 2-ethyl hexanol (as oil phase) and span-80 as surfactant. IKA T25 digital ultra turrax, a high speed homogenizer was used to homogenize all components at a speed of 22000 rpm in a vessel and temperature is maintained at $40^{\circ} \mathrm{C}$ in a water bath.Aqueous solution of sodium borohydride was added drop wise (2-3 drop/sec.) in the mixture under high shear mixing conditions. A spontaneous reduction reaction occurred due to exothermic nature of reaction. Formation of black colloidal suspension reveals the formation of silver nanoparticles.

A reaction between silver nitrate and sodium borohydride in water solution could be described by following chemical reaction ${ }^{15}$ :

$$
\begin{array}{lr}
\mathrm{AgNO}_{3}+\mathrm{NaBH}_{4} \rightarrow \mathrm{Ag}+1 / 2 \mathrm{H}_{2}+1 / 2 \mathrm{~B}_{2} \mathrm{H}_{6}+\mathrm{NaNO}_{3} \\
\mathrm{~B}_{2} \mathrm{H}_{6}+\mathrm{H}_{2} \mathrm{O} \rightarrow 6 \mathrm{H}_{2}+2 \mathrm{H}_{3} \mathrm{BO}_{3} & \ldots(1) \\
\mathrm{NaBH}_{4}+2 \mathrm{H}_{2} \mathrm{O} \rightarrow \mathrm{NaBO}_{2}+4 \mathrm{H}_{2} & \ldots(2) \\
\mathrm{BH}_{4}^{-}+\mathrm{H}_{3} \mathrm{O}^{+}+2 \mathrm{H}_{2} \mathrm{O} \rightarrow \mathrm{H}_{3} \mathrm{BO}_{3}+4 \mathrm{H}_{2} & \ldots(3)
\end{array}
$$

Alongwith reaction (1), simultaneous reaction $(3,4)$ and coupled reaction( 2 ) could proceed in a water environment ${ }^{16}$. A decrease in $\mathrm{pH}$ value is resulted from the formation of boric acid. According to Song et al. ${ }^{4}$, metal reduction generally proceeds according to the following reaction:

$\mathrm{Ag}^{+}+\mathrm{BH}_{4}^{-}+3 \mathrm{H}_{2} \mathrm{O} \rightarrow \mathrm{Ag}+\mathrm{B}(\mathrm{OH})_{3}+3.5 \mathrm{H}_{2}$

Taking into consideration reaction (1) proposed by Bruzzoniti et al. ${ }^{15}$, one product (diborane) is absent in reaction (5).

\section{Characterization of silver nanoparticles}

The silver nanoparticles are characterized for size and shape by TEM analysis using FEI Tecnai G2 F20 X-TWIN transmission electron microscope. Particle size distribution is taken with the help of
Malvern Zeta sizer ZS90 instrument. XRD analysis is done by $\mathrm{X}$-ray diffractometer, model ISO Debyeflex 2002.

\section{RESULTS AND DISCUSSIONS}

\section{Effect of Ag lon concentration}

The variation in average particle size of silver nanoparticles with concentration of silver nitrate along with DLS particle size distribution histograms is shown in fig.1. Hereit was observed that the size of silver nanoparticles increased with the increase insilver nitrate concentration. This is attributed to increase in the number of nuclei formed on increasing concentration ${ }^{17}$.

The average diameter of silver nanoparticles obtained with $0.05 \mathrm{M}, 0.08 \mathrm{M}$ and $0.1 \mathrm{M}$ concentration of silver nitrate were $19.13 \mathrm{~nm}, 16.29 \mathrm{~nm}$ and $68.86 \mathrm{~nm}$ respectively. On increasing the $\mathrm{AgNO}_{3}$ concentration from $0.05 \mathrm{M}$ to $0.08 \mathrm{M}$, the nuclei formation rate increased more significantly than the growth rate of silver nanoparticles and it led to large amount of small nanoparticles, which absorb span80 (taken as $4.5 \mathrm{mmol}$ ), thus preventing any further agglomeration ${ }^{18}$. On increasing $\mathrm{AgNO}_{3}$ concentration further up to $0.1 \mathrm{M}$, the collision frequency of silver nanoparticles increased significantly. Thus protection by span-80 obtained on silver nanoparticles surface by adsorption of span-80 molecules was weakened and small nanoparticles could aggregate into larger particles and attained different shapes as well as spherical ones. Thus a transition in morphology from spherical to triangular shape is observedonincreasing concentration of $\mathrm{AgNO}_{3}$ up to 0.1M. However, it was observed that further increase in concentration of silver salt did not cause very much increment in size.

A typical XRD pattern of the silver nanoparticles prepared at $0.08 \mathrm{M}$ concentration of silver nitrate (sample b-2 in fig. 1 ) is shown in fig. 2 . The X-ray powder diffraction (XRD) pattern was recorded with $\mathrm{Cu}$ Ká radiation $\left(\lambda=1.54184 \mathrm{~A}^{0}\right)$ in 2-theta ranging from $10^{\circ}$ to $100^{\circ}$ (scan speed of $3 \% \mathrm{~min})$. Here, the Bragg's reflections observed at 2-theta values of $38.115,44.229,64.443,77.397$ and 81.541 representing [111], [200], [220], [311] and [222] planes respectively; which indicate the formation of silver nanocystals with cubic face 


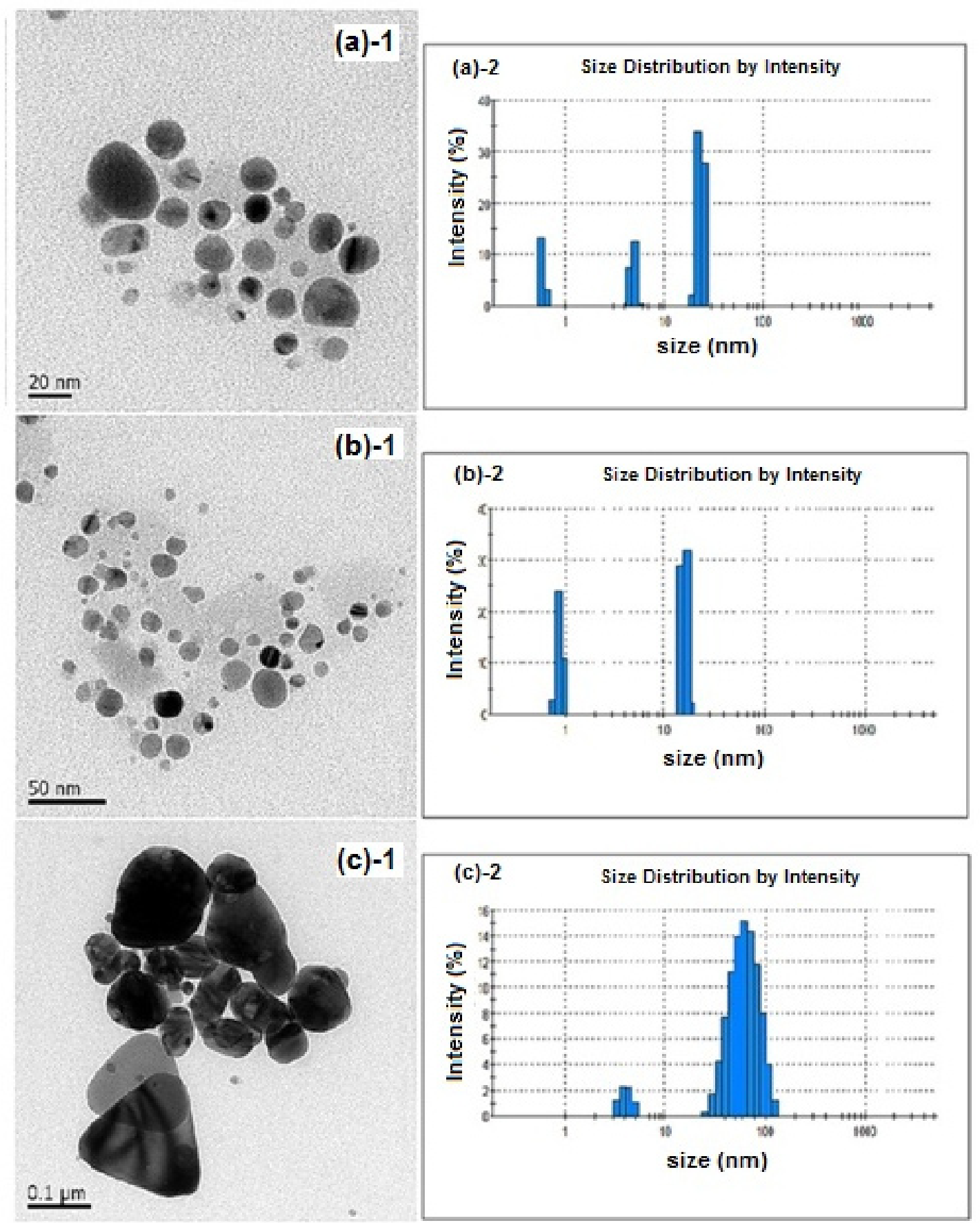

Fig. 1: (TEM images and DLS size distribution histograms of silver nanoparticles synthesized with different concentration of silver nitrate: (a)-1 and (a)-2 at 0.05M, (b)-1 and (b)-2 at 0.08M, (c)-1 and (c)-2 at $0.10 \mathrm{M}$.) 
centered (FCC) structure. The peaks obtained are consistent with JCPDS card no. 87-0597. Since any other peak was not observed, it indicates the purity of silver nanoparticles.

\section{Effect of concentration of reducing agent}

In the proposed synthesis process, concentration of reducing agent was found as an important factor which affects the silver nanoparticle size (Fig.3). An increment in the concentration of the $\mathrm{NaBH}_{4}$ was carried out w.r.to constant concentration of silver salt $(0.08 \mathrm{M})$, in a simple multiple ratio as $0.08 \mathrm{M}, 0.16 \mathrm{M}, 0.24 \mathrm{M}$ and $0.32 \mathrm{M}$. It was observed from particle size distribution histograms of silver nanoparticles that, at low conc. of $\mathrm{NaBH}_{4}$ (e.g. $0.08 \mathrm{M}$ and $0.16 \mathrm{M}$ ) only few and not all $\mathrm{Ag}^{+}$ions were reduced (Fig.3a, 3b). Thus overlapping of nucleation and growth process, which is more pronounced as the slower chemical reaction, led to larger and more polydisperse final nanoparticles sizes. The average diameter of the silver nanoparticles at $0.08 \mathrm{M}$ and $0.16 \mathrm{M}$ concentration of $\mathrm{NaBH}_{4}$ was observed as $45.33 \mathrm{~nm}$ and $83.80 \mathrm{~nm}$. At very high conc. of $\mathrm{NaBH}_{4}(0.32 \mathrm{M})$, the anion (i.e. $\mathrm{BH}_{4}^{-}$) occupancy per reverse micelle increased (Fig.3d). This can promote intermicellar nucleation and growth, leading to increase in the average particle size and polydispersity ${ }^{2}$. The average diameter of the silver nanoparticles here observed as $296.29 \mathrm{~nm}$. At an intermediate conc. of $\mathrm{NaBH}_{4}$ (i.e. $0.24 \mathrm{M}$ ), both nucleation and growth process were so controlled, that nearly monodisperse particle size distribution histogram (fig.3c) was obtained. At this optimum concentration of reducing agent (i.e. 0.24M), nucleation was faster when the chemical reaction was faster. Since growth is strongly influenced by the nuclei number already formed at a given time, a great number of nuclei favor a fast autocatalytic growth, giving rise to a large number of small nanoparticles ${ }^{19}$. The average diameter of the silver nanoparticles at this concentration was observed as $20.42 \mathrm{~nm}$. TEM monograph of silver nanoparticles synthesized at $0.24 \mathrm{M}$ conc. of $\mathrm{NaBH}_{4}$ is shown in fig.3e which was in accordance with the particle size distribution pattern obtained here.

\section{Effect of concentration of Surfactant}

In view of all the parameters related to experiment, when concentration of span- 80 decreased keeping allother parameters constant [conc. of silver salt $(0.08 \mathrm{M})$, conc. of reducing agent $(0.24 \mathrm{M})$ and 2-ethyl hexanol (10\% by volume)], it was observed that average size and polydispersity of silver nanoparticles increased. Since our proposed synthesis procedure utilizes high shear mixing of bulk water phase $(250 \mathrm{ml})$ and 2-ethyl hexanol $(10 \%$ by volume), variation in the concentration of surfactant was done on the basis of ratios $\left(F_{S}\right)$, which was ratio of 2-ethyl hexanol $(\mathrm{ml})$ tospan-80(ml). An increase in $\mathrm{F}_{\mathrm{s}}$ value was associated with decrease in amount of span-80. Six ratios (Fs) have been studied viz, $\mathrm{Fs}=8,10,12,15,20$ and 30 . The variations in particles distribution for different ratios $\left(F_{\mathrm{S}}\right)$ are given in fig.4. The average diameter of the silver

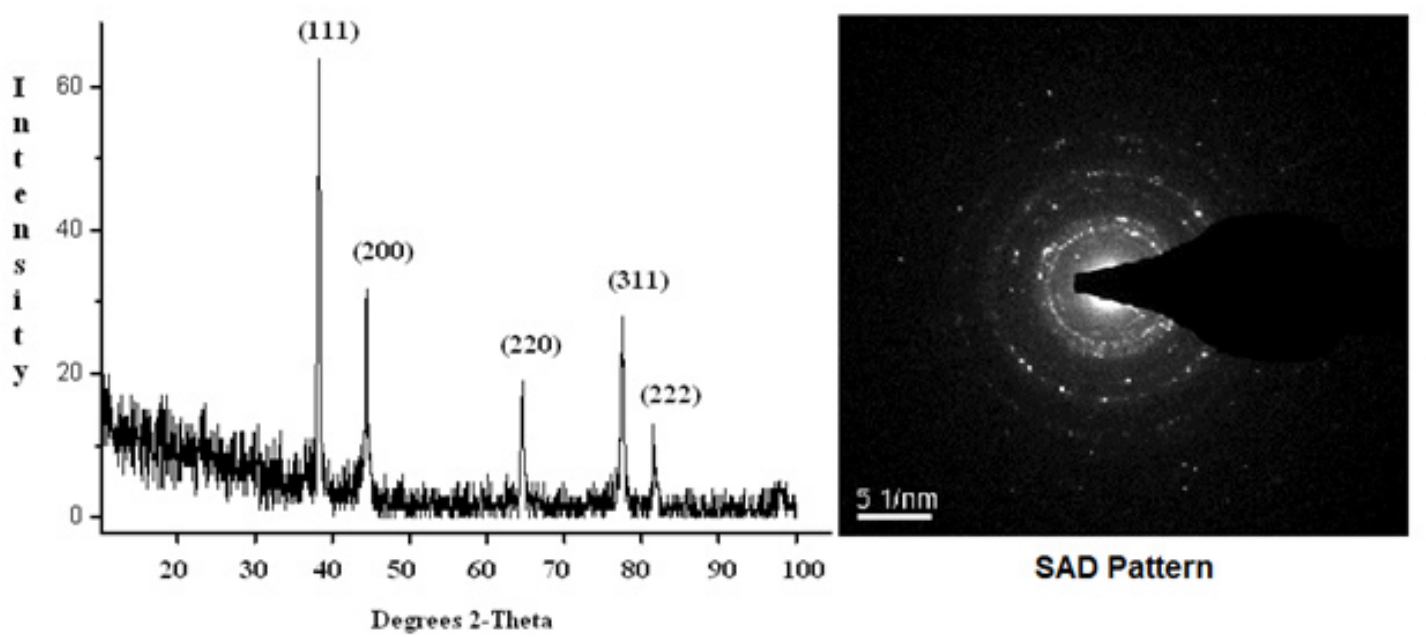

Fig. 2: (XRD and SAD pattern of silver nanoparticles synthesized with $0.08 \mathrm{M} \mathrm{AgNO}_{3}$ solution.) 
nanoparticles synthesized at above mentioned ratios obtained as $13.02 \mathrm{~nm}, 15.62 \mathrm{~nm}, 25.10 \mathrm{~nm}$, $65.36 \mathrm{~nm}, 133.28 \mathrm{~nm}$ and $300.12 \mathrm{~nm}$, respectively. Therefore, tremendous increase in particle size with polydispersity was observed on decreasing the amount of surfactant.

This could be possibly as microemulsion consists of nanometer size water droplets which are dispersed in a continuous oil medium and stabilized by surfactant molecules accumulated in the oil-water interface. The main function of the droplet nanoreactor is to provide a compartmentalized medium in order to prevent phase separation of the particles ${ }^{20,21}$. An increase in the concentration of span-80 produced large number of span-80 reverse micelles. Hence, solubilization capacity of the reactants in the reverse micelle increased in conjunction with a decrease in the radii of the reverse micelle. These resulted in an increase in the amount of nanoparticles formed and a simultaneous decrease in the average size of the silver nanoparticles.

\section{Effect of content of oil phase}

The variation in oil phase content (i.e. 2-ethyl hexanol) was done and it was taken at the levels of $5 \%, 10 \%, 15 \%, 20 \%$ and $30 \%$ by volume, keeping all other parameters [conc. of silver salt $(0.08 \mathrm{M})$, conc. of reducing agent $(0.24 \mathrm{M})$ and surfactant (6.44 mmol)] constant. Effect of increasing

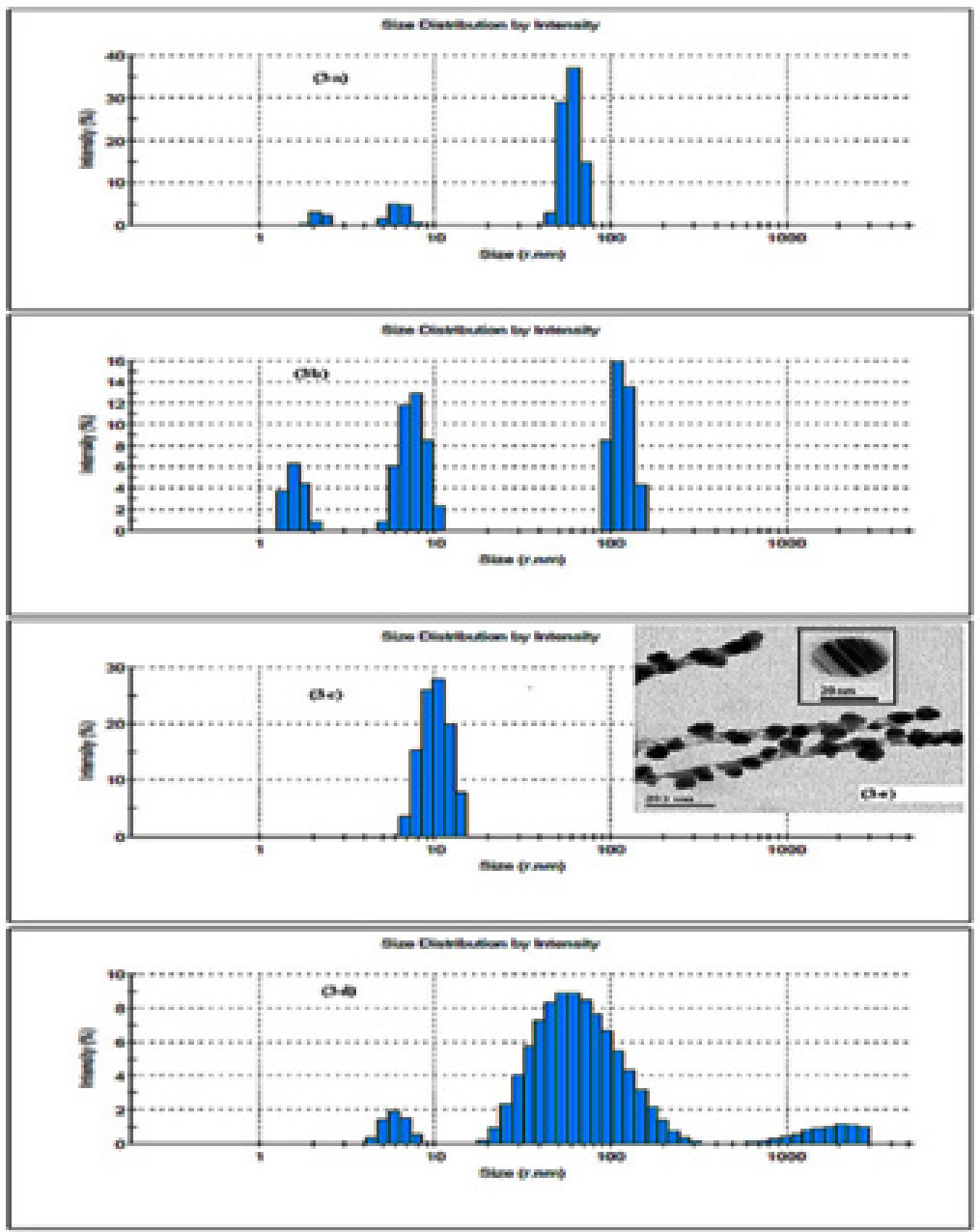

Fig. 3: DLS size distribution histograms of silver nanoparticles synthesized at different concentration of $\mathrm{NaBH}_{4}$ : (3a) $0.08 \mathrm{M}$, (3b) $0.16 \mathrm{M},(3 \mathrm{c}) 0.24 \mathrm{M}$, (3d) $0.32 \mathrm{M}$ and (3e) TEM image of silver nanoparticles prepared at $0.24 \mathrm{M} \mathrm{NaBH}$ 
oil phase content on size and distribution of silver nanoparticles is shown in fig.5. It was found that, an increase in the oil phase content led to increase in the averagesize of the silver nanoparticles. The average size of the silver nanoparticles at $5 \%, 10 \%$, $15 \%, 20 \%$ and $30 \%$ (by volume) oil phase content was observed to be $22.98 \mathrm{~nm}, 23.46 \mathrm{~nm}, 135.98 \mathrm{~nm}$, $157.30 \mathrm{~nm}$ and $193.84 \mathrm{~nm}$, respectively. This could be explained asat an optimum value of oil phase content, with the continuous addition of aqueous solution of $\mathrm{NaBH}_{4}$ up to $\mathrm{pH}=9$ under high shear mixing condition, phase inversion of microemulsion occurs. In our study, during phase inversion conditions a lot of physical changes occurred including change in particle size. By successively addition of $\mathrm{NaBH}_{4}$,
$\mathrm{pH}$ of the medium increased and a transition in the spontaneous radius of curvature of span-80 is observed. At this time system crosses a point of zero spontaneous curvature and minimal surface tension, promoting the formation of finely dispersed droplets $^{22}$. At higher oil phase content (i.e. more than $10 \%$ by volume), phenomenon of phase inversion occurred after a long time and relatively a large amount of aqueous solution of $\mathrm{NaBH}_{4}$ consumed up to $\mathrm{pH}=9$. Evidently avg. particle size with polydisperse nature increased. At a very low oil phase content $(5 \%$ by volume), the content of continuous phase is very small w.r.to surfactant (span-80), therefore phase inversion occurred very quickly with the addition of aq. $\mathrm{NaBH}_{4}$. Thus silver nanoparticles obtained here

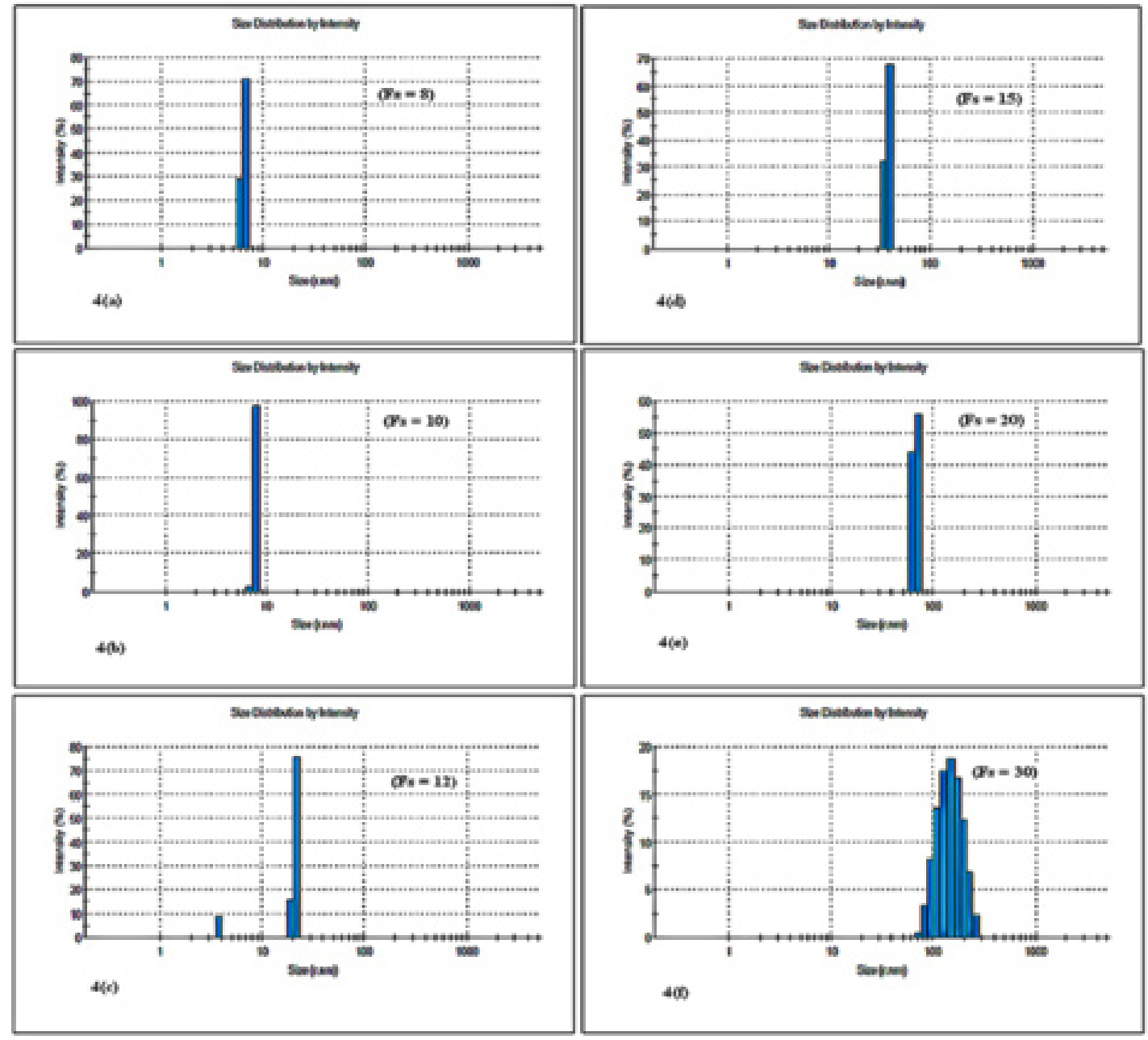

Fig. 4: DLS size distribution histograms of silver nanoparticles synthesized at different $F_{s}$ ratios (i.e. amount of organic phase/amount of surfactant): 4(a) $F_{s}=8,4(b) F_{s}=10,4(c) F_{s}=12$, 4(d) $F_{s}=15$, $4(e) F_{s}=20$ and $4(f) F_{s}=30$ 

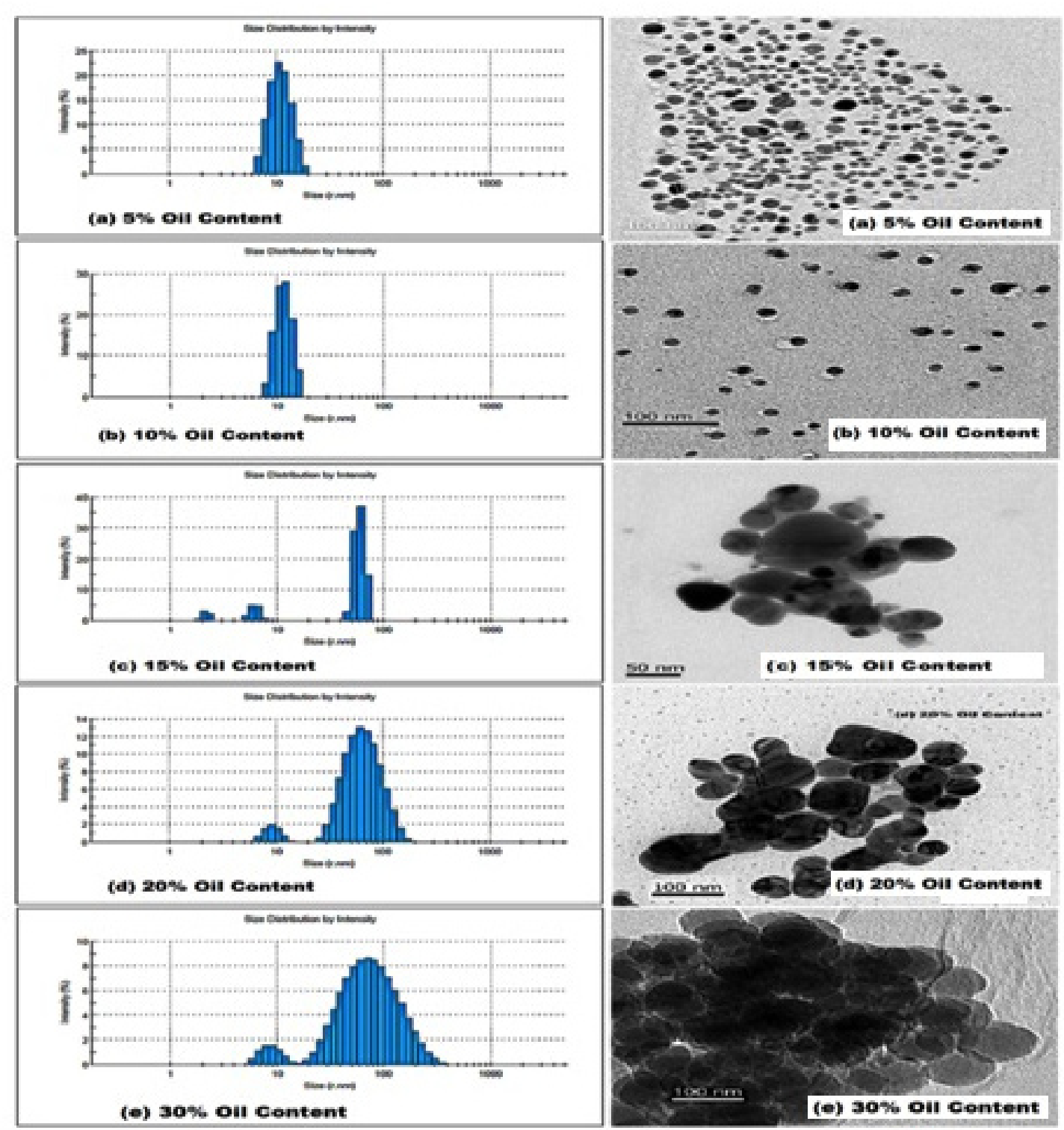

Fig. 5: DLS size distribution histograms of silver nanoparticles synthesized by taking different amount of organic phase (\% by volume): (a) $5 \%$, (b) $10 \%$, (c) $15 \%$, (d) $20 \%$ and (e) $30 \%$

show a little bit high particle size distribution from 6-20 $\mathrm{nm}$ due to insufficient adsorption by surfactant molecules.

\section{CONCLUSION}

Silver nanoparticles with uniform particle size distribution were successfully synthesized by carrying out a chemical reduction reaction in a microemulsion media, prepared under high shear mixing conditions. Aqueous solution of silver nitrate was taken as silver ion source, sodium borohydride as reducing agent, 2- ethyl hexanol as organic phase and span-80 as stabilizer. The effect of silver ion concentration, strength of reducing agent, amount of surfactant and content of oil phase (i.e. organic phase), on size and size distribution of silver nanoparticles were investigated. It was found that size of silver nanoparticles increased on increasing silver nitrate concentration; also a 
deviation in morphology from spherical to triangular shape is observed. Concentration of reducing agent was found to play a key role in this reaction process, a sharp increase in size is observed on increasing conc. of reducing agent. Stabilization of synthesized silver nanoparticles by adsorption of span-80 surfactant showed that decrease in amount of surfactant w.r.to continuous oil phase led to increase in avg. particle size of silver nanoparticles. Amount of oil phase content is also found as important parameter in tuning the synthesis process for smaller size silver nanoparticles with narrow size distribution.
The synthesized silver nanoparticles were successfully characterized via TEM and DLS analysis for their size, shape and size distribution. XRD analysis showed their good crystalline nature. Thus silver nanoparticles synthesized by this process have an extensive application prospects and can be preserved for a long time.

\section{ACKNOWLEDGMENTS}

The authors are thankful to IIT, Kanpur and NST Division (DMSRDE) for the support in the characterization of the samples.

\section{REFERENCES}

1. Sobczak-Kupiec, A.; Malina, D.; Wzorek, Z.; Zimowska, M. Micro and Nano Letters. 2011, 6, 656-660

2. Solanki, J. N.; Murthy, Z.V. P. Colloid and Surfaces A: Physicochemical Engineering Aspects. 2010, 359, 31-38

3. Ramaswamy, V.; Vimalathithan, R.M.; Ponnusamy, V. Advances in Applied Science Research. 2010, 1, 197

4. Song, K. C.; Lee, S. M.; Park, T. S.; Lee, B. S. Korean Journal of Chemical Engineering. 2009, 26, 153-155

5. Goia, D.V.; Matijevic, E.; New Journal of Chemistry. 1998, 98, 1203-1215

6. Zhang, W.; Qiao, X.; Qiu, X. Journal of Matterials Science. 2009, 44, 1076-1081

7. Rai, M.; Yadav, A.; gade, A. Biotechnology Advances. 2009, 27, 76-83

8. Nourafkan, E.; Alamdari, A. Journal of Industrial and Engineering Chemistry. 2014, 20, 3639-3645

9. Khadzhiev, Salambek N.; Kadiev, Khusain M.; Yampolskaya, Galina P.; Kadieva, Malkan Kh. Advances in Colloid and Interface Science. 2013, 197-198, 132-145

10. McClements, DJ.; Li, Y. Advances in Colloid and Interface Science. 2010, 159, 213-228

11. Bilbao Sainz, C.; AvenaBustillos, RJ.; Wood, DF.; Williams, TG.; Mchugh, TH. Journal of Agricultural and Food Chemistry. 2010, 58, 11932-11938
12. Jahanzad, F.; Josephides, D.; Mansourian, A.; Sajjadi, S. Industrial and Engineering Chemistry Research. 2010, 49, 7631-7637

13. Khan, Z.; Al-Thabaiti, S. A.; Obaid, A. Y.; Al-Youbi, A. O. Colloids and Surface B: Biointerfaces. 2011, 82, 513-517

14. Katiyar, A.; Singh, A. N.; Shukla, P.; Nandi, T. Powder Technology. 2012, 224, 86-89

15. Bruzzoniti, M. C.; Kobylinska, D. K.; Franko, M.; Sarzanini, C. Analytica Chimica Acta. 2010, 665, 69-73

16. Demirci, U. B.; Miele, P. Comptes Rendus Chimie. 2009, 12, 943-945

17. Bae, D. S.; Kim, E. J.; Bang, J. H.; Kim, S. W.; Han, K. S.; Lee, J. K.; Kim, B. I.; Adair, J. H. Metals and Materials International. 2005, 11, 291

18. Zhang, W.; Qiao, X.; Chen, J.; Wang, H. Journal of Colloid and Interface Science. 2006, 302, 370-373

19. de Dios, M.; Barroso, F.; Tojo, C.; Blanco, M. C.; Lopez-Quintela, M. A. Colloid and Surfaces A: Physicochemical Engineering Aspects. 2005, 270-271, 83-87

20. Capek, I. Advances in Colloid and Interface Science. 2004, 10, 49-74

21. Tojo, C.; de Dios, M.; Barroso, F. Materials. 2011, 4, 55-72

22. Ee, SL.; Duan, X.; Liew, J.; Nguyen, QD. Chemical Engineering Journal. 2008, 140, 626-631. 